Nonlinear Processes in Geophysics (2002) 9: 87-99

Nonlinear Processes

in Geophysics

(C)European Geophysical Society 2002

\title{
Nonlinear waves and solitons propagating perpendicular to the magnetic field in bi-ion plasma with finite plasma pressure
}

\author{
E. M. Dubinin ${ }^{1,3}$, K. Sauer ${ }^{1}$, J. F. McKenzie ${ }^{1,2}$, and G. Chanteur ${ }^{3}$ \\ ${ }^{1}$ Max-Planck Institut für Aeronomie, Katlenburg-Lindau, Germany \\ ${ }^{2}$ School of Pure and Applied Physics, University of Natal, Durban, South Africa \\ ${ }^{3}$ Centre d'Etude des Environnements Terrestre et Planetaires, Velizy, France
}

Received: 25 June 2001 - Revised: 16 October 2001 - Accepted: 6 November 2001

\begin{abstract}
We investigate the nature of nonlinear waves propagating transverse to the magnetic field in a bi-ion plasma including plasma pressure. By using the conservation laws derived from the multi-ion fluid equations the system may be described by a single order differential equation whose properties control the structure of the flow and the magnetic field. Compressive solitons exist in specific ranges of the characteristic Mach numbers. Various features of solitons differ in different existence "windows". For example, there are solitons that contain a strong proton rarefaction core embedded in the main compressional structure. Compressive solitons are found in a wide range of flow parameters. Finite ion pressure introduces critical Mach numbers. In contrast to a plasma consisting only of protons and electrons these singular points are reached where a specific combination of ion and electron speeds lies on particular locii, in multi-parameter space, which corresponds to the generalized "sonic point" of the compound system.
\end{abstract}

\section{Introduction}

The multi-ion nature of space plasmas gives rise to new interesting effects which have not been present in a "classical" proton-electron plasma. A tractable model for describing the behaviour of a bi-ion plasma has been developed, for example, by Sauer et al. (1994), using multi-fluid equations. The existence of a second ion population leads to an additional coupling between the ions and electrons through the Lorentz force and charge neutrality. Even a small admixture of heavy ions into a proton-electron plasma may significantly modify the plasma wave and flow properties. In particular, the appearance of new additional wave modes leads to new types of stationary nonlinear structures.

Recently, McKenzie et al. (2001a) studied stationary structures streaming transverse to the magnetic field in a cold bi-

Correspondence to: E. M. Dubinin

(dubinin@linmpi.mpg.de) ion plasma. It was shown that the stationary solutions are governed by a second order differential equation. Examples of numerical solutions for transverse solitons in a cold bi-ion plasma have been given by Sauer et al. (2000), McKenzie et al. (2001a).

In this paper we examine the structure of stationary waves including the effect of plasma pressure which manifests itself through the proton $\left(M_{p}\right)$ and heavy ion $\left(M_{h}\right)$ Mach numbers. The inclusion of electron pressure does not change the qualitative structure of compressional waves since the Alfvén Mach number is simply replaced by the "fast Mach number". The inclusion of proton and heavy ion pressures affects the structure significantly because ion pressures become important reservoirs in momentum exchanges between ion fluids and the magnetic field. It is shown that by using the conservation laws the problem can be reduced to a first order differential equation. Another important issue is the appearance of "critical points". In a bi-ion plasma such points or singularities are reached on specific locii of the speeds of the protons, heavy ions and electrons, and can be shown to correspond to the generalization of the idea of a sonic point of the compound system (McKenzie et al., 1993).

The layout of the paper is as follows. The governing equations for a bi-ion plasma given in Sect. 2 are the usual fluid equations for each species which are coupled together through the Lorentz forces and charge neutrality. Since electron inertia is neglected, the electric field may be eliminated and the magnetic is frozen into the electron fluid. Thus, the equations may be regarded as an extension to a bi-ion fluid of the Hall-MHD description of a single ion - electron system. In Sect. 3 we derive the constants of the motion for the special case of flow transverse to the magnetic field and show that by using them the second order differential equation, which describes the system, can be reduced to a first order differential equation. In Sect. 4 we discuss the main features of stationary solutions by using the properties of the "momentum hodograph" which provides the relations between the speeds of the species implied by conservation of total momentum and the charge neutrality constraint. We also present 
examples of phase trajectories of the system in the planes $\left(d u_{i x} / d x, u_{i x}\right)\left(u_{i x}\right.$ are the speeds of the ion and electron fluids), along with the corresponding numerical stationary solutions. To better illustrate the main effects arising from the coupling between the electron and ion fluids, we consider the case where the abundance of heavy ions is rather high $\left(\alpha=n_{h o} / n_{p o}=0.2\right.$, where $\alpha$ is the density ratio between the protons and the heavy ions) and the total mass flux is dominated by the heavy ions $\left(m_{h} / m_{p}=15\right.$, here $m_{p, h}$ are masses of protons and heavy ions). In Sect. 5 we analyze the structure equation in detail and consider the main features introduced by the effects of finite plasma pressure. It is shown that soliton structures can be constructed in different ranges of characteristic Mach numbers in which the properties of these solutions are different. Some transitions comprise of a compression followed by a rarefaction core. The amplitudes of the stationary waves are expressed as a function of the Mach numbers. Section 6 addresses the problem of the critical Mach numbers and critical amplitudes of solitons in a bi-ion plasma.

\section{Governing equations}

In a magnetized plasma consisting of protons $(p)$, heavy ions $(h)$ and electrons $(e)$, the fluid equations for each constituent are

$\frac{\partial n_{i}}{\partial t}+\nabla \cdot\left(n_{i} \boldsymbol{u}_{i}\right)=0$,

$m_{i} n_{i} \frac{D_{i} \boldsymbol{u}_{i}}{D t}=e n_{i} q_{i}\left(\boldsymbol{E}+\boldsymbol{u}_{i} \times \boldsymbol{B}\right)-\nabla p_{i}$,

where $m_{i}$ is the mass $(i=p$, protons, $i=e$, electrons, $i=h$, heavy ions), $q_{i}$ the ion charge, $n_{i}$ the number density, $p_{i}$ the pressure of each species, $\boldsymbol{B}$, and $\boldsymbol{E}$ are the magnetic and electric field, respectively, and the convective derivative is

$$
\frac{D_{i}}{D t}=\frac{\partial}{\partial t}+\boldsymbol{u}_{i} \cdot \nabla
$$

If electron inertia is negligible, the electric field is given by

$$
\boldsymbol{E}=-\boldsymbol{u}_{e} \times \boldsymbol{B}-\frac{\nabla p_{e}}{n_{e} e} .
$$

Faraday's law becomes

$$
\frac{\partial \boldsymbol{B}}{\partial t}=\operatorname{curl}\left(\boldsymbol{u}_{e} \times \boldsymbol{B}\right),
$$

which shows that the magnetic field is frozen into the electron fluid. The remaining equation, Ampere's law, is

curl $\boldsymbol{B}=\mu_{o} \boldsymbol{j}$,

in which we neglect the displacement current and the current $\boldsymbol{j}$ is given by

$\boldsymbol{j}=\sum_{i=p, h} e q_{i} n_{i} \boldsymbol{u}_{i}-e n_{e} \boldsymbol{u}_{e}$.
This is the standard MHD description, except that $\boldsymbol{B}$ is frozen into the electrons which allows for dispersive Hall current effects. The existence of a second ion population leads to an additional coupling between all the species. This coupling is readily revealed by eliminating $\mathbf{u}_{e}$ from Eq. (4) and using Eqs. (6) and (7), to obtain

$\boldsymbol{u}_{e}=-\frac{\nabla \times \boldsymbol{B}}{\mu_{o} n_{e} e}+\frac{n_{p} \boldsymbol{u}_{p}}{n_{e}}+\frac{q n_{h} \boldsymbol{u}_{h}}{n_{e}}$.

Upon using the charge neutrality constraint $n_{e}=n_{p}+q n_{h}$, the momentum equations for the protons and the heavy ions may then be written in the form

$$
\begin{gathered}
m_{p} n_{p} \frac{D_{p} \boldsymbol{u}_{p}}{D t}=\frac{e q n_{p} n_{h}}{n_{e}}\left[\left(\boldsymbol{u}_{p}-\boldsymbol{u}_{h}\right) \times \boldsymbol{B}\right] \\
+\frac{n_{p}}{\mu_{o} n_{e}}(\nabla \times \boldsymbol{B}) \times \boldsymbol{B}-\frac{n_{p}}{n_{e}} \nabla p_{e}-\nabla p_{p}, \\
m_{h} n_{h} \frac{D_{h} \boldsymbol{u}_{h}}{D t}=\frac{e q n_{p} n_{h}}{n_{e}}\left[\left(\boldsymbol{u}_{h}-\boldsymbol{u}_{p}\right) \times \boldsymbol{B}\right] \\
+\frac{n_{h}}{\mu_{o} n_{e}}(\nabla \times \boldsymbol{B}) \times \boldsymbol{B}-\frac{n_{h} q}{n_{e}} \nabla p_{e}-\nabla p_{h} .
\end{gathered}
$$

The main new feature which appears in these equations, as compared to the standard MHD equations, is the Lorentz force term arising from the differential streaming between the ion fluids, which has the opposite action $( \pm$ sign) on the protons and the heavy ions. Relative streaming arises due to the difference in inertia which, in turn, leads to the generation of the motional electric fields in the reference frames of both streaming ion fluids and therefore results in a strong coupling between the ions.

\section{Conservation laws and the structure equation}

Here we consider adiabatic stationary $\left(\frac{\partial}{\partial t}=0\right)$ structures with variations perpendicular to the magnetic field and flow transverse to $\boldsymbol{B}\left(\boldsymbol{B}=(0,0, B), \mathbf{u}_{i}=\left(u_{i x}, u_{i y}, 0\right)\right.$, so that $\frac{D}{D t}=u_{i x} \frac{d}{d x}$ for variations in $x$ only. The continuity equations become

$n_{i} u_{i x}=f_{i}$, const.

Adding the $x$-components of the momentum Eq. (2) for ions and electrons (with $m_{e}=0$ ) and then integrating yields the total momentum conservation equation in the $x$-direction,

$\sum_{i=p, h} m_{i} f_{i} u_{i x}+\frac{B^{2}}{2 \mu_{o}}+\sum_{i=p, h, e} p_{i}=$ const.

Faraday's law tell us that $E_{y}=$ const and $E_{z}=$ const. Using Eq. (4) we have

$E_{y}=u_{e} B=$ const, $E_{z}=0$.

For adiabatic flows we have

$p_{i} \propto n_{i}^{\gamma_{i}} \propto u_{i}^{-\gamma_{i}}$. 
Equations (10) to (13) represent the important conserved quantities of number flux, momentum flux, transverse electric field and entropy. For convenience, we take $\gamma_{i}=2$ and assume that at $x=-\infty \quad n_{p}=n_{p o}=1, n_{h}=n_{h o}=\alpha$, $u_{i x}=u_{o}=1$, and $B=B_{o}=1$ with the result that the normalized equations become

$n_{p} u_{p}=f_{p}=1, n_{h} u_{h}=f_{h}=\alpha$,

$$
\begin{gathered}
u_{p x}-1+\alpha \mu\left(u_{h x}-1\right)+\frac{1}{2 M_{f}^{2}}\left(\frac{1}{u_{e x}^{2}}-1\right)+ \\
\frac{1}{2 M_{p}^{2}}\left(\frac{1}{u_{p x}^{2}}-1\right)+\frac{\alpha \mu}{2 M_{h}^{2}}\left(\frac{1}{u_{h x}^{2}}-1\right)=0,
\end{gathered}
$$

where $\mu=m_{h} / m_{p}$ and $M_{f}, M_{p}, M_{h}$ are the Mach numbers of the plasma flow at $x=-\infty$,

$M_{f}^{2}=\frac{u_{o}^{2}}{V_{A p}^{2}+c_{e}^{2}}, M_{p}^{2}=\frac{u_{o}^{2}}{c_{p}^{2}}, M_{h}^{2}=\frac{u_{o}^{2}}{c_{h}^{2}}$.

Here $V_{A p}=B_{o} /\left(\mu_{o} n_{p o} m_{p}\right)^{1 / 2}$ is the Alfvén speed based on the proton mass density, and $c_{p}=\left(\gamma_{p} p_{p o} / n_{p o} m_{p}\right)^{1 / 2}, c_{h}=$ $\left(\gamma_{h} p_{h o} / n_{h o} m_{h}\right)^{1 / 2}, c_{e}=\left(\gamma_{e} p_{e o} / n_{e o} m_{p}\right)^{1 / 2}$, are the sound speeds, with the suffix "o" referring to values at $x=-\infty$.

Adding the $y$-components of the momentum equations for the ions and by using $j_{x}=e\left(f_{p}+q f_{h}-f_{e}\right)=0$, we obtain the normalized momentum conservation equation in the $y$ direction,

$u_{p y}+\alpha \mu u_{h y}=0$.

The differential equation which determines the structure of the transition for stationary flows in a bi-ion plasma is a highly nonlinear second order differential equation. It follows that subtracting the $x$-components of the equations of motion for protons and heavy ions, and eliminating the transverse electron velocity $u_{e y}$, yields

$$
\begin{aligned}
q u_{p x} & \left(1-\frac{u_{p x o}^{3}}{M_{p}^{2} u_{p x}^{3}}\right) \frac{d u_{p x}}{d x}+\mu u_{h}\left(\frac{u_{h o}^{3}}{M_{h}^{2} u_{h x}^{3}}-1\right) \frac{d u_{h}}{d x} \\
= & \frac{e q}{m_{p}}\left(u_{p y}-u_{h y}\right) \frac{u_{e x o} B_{o}}{u_{e x}},
\end{aligned}
$$

by normalizing all variables to their values at $x=-\infty\left(u_{i y}\right.$ is normalized to $u_{i o}$ ), and using the length scale $u_{o} / \Omega_{p}$, where $\Omega_{p}=e B_{o} / m_{p}$ is the proton gyrofrequency:

$$
\begin{aligned}
q(1 & \left.-\frac{1}{M_{p}^{2} u_{p x}^{3}}\right) u_{p} \frac{d u_{p}}{d x}+\mu\left(\frac{1}{M_{h}^{2} u_{h x}^{3}}-1\right) u_{h x} \frac{u_{h x}}{d x} \\
& =\frac{q\left(u_{p y}-u_{h y}\right)}{u_{e x}} .
\end{aligned}
$$

Differentiating Eq. (19) with respect to $x$ and using

$$
\frac{d}{d x}\left(u_{p y}-u_{h y}\right)=\frac{u_{e x}-u_{p x}}{u_{p x} u_{e x}}-\frac{q}{\mu} \frac{u_{e x}-u_{h x}}{u_{h x} u_{e x}},
$$

we obtain a second order differential equation in which the terms containing the derivatives $d u_{h x} / d x$ and $d u_{e x} / d x$ can be replaced by terms with $d u_{p x} / d x$ by differentiating the momentum conservation Eq. (15) and the equation $j_{x}=0$. The latter may be written in the form:

$u_{p x} u_{h x}(1+\alpha q)-u_{e x}\left(u_{h x}+\alpha q u_{p x}\right)=0$.

However, the second order equation can be reduced to a first order differential equation if we use an additional conservation law (conservation of energy flux), which can be obtained mathematically in the following way. Multiplying the equation for the $x$-component of motion for the protons (heavy ions) by $u_{p x}\left(u_{h x}\right)$ and adding them using

$e E_{x}=-e u_{e y} B-\frac{1}{n_{e}} \frac{d p_{e}}{d x}$

and Ampere's law

$-\frac{1}{\mu_{o} e} \frac{d B}{d x}=n_{p} u_{p y}+n_{h} q u_{h y}-n_{e} u_{e y}$,

we obtain

$$
\begin{aligned}
& \frac{m_{p} f_{p}}{2} \frac{d u_{p x}^{2}}{d x}+\frac{m_{h} f_{h}}{2} \frac{d u_{h x}^{2}}{d x}+u_{e x} \frac{d}{d x}\left[\frac{B^{2}}{2 \mu_{o}}+p_{e}\right] \\
& +u_{p x} \frac{d p_{p}}{d x}+u_{h x} \frac{d p_{h}}{d x}=e f_{p} u_{p y}\left(1-\frac{u_{e x}}{u_{p x}}\right) B \\
& +e q f_{h} u_{h y}\left(1-\frac{u_{e x}}{u_{h x}}\right) B .
\end{aligned}
$$

In a similar way, multiplying the equation for the $y$ component of the motion for the protons (heavy ions) by $u_{p y}\left(u_{h y}\right)$ and adding them, we obtain

$$
\begin{aligned}
& \frac{m_{p} f_{p}}{2} \frac{d u_{p y}^{2}}{d x}+\frac{m_{h} f_{h}}{2} \frac{d u_{h y}^{2}}{d x}=-e f_{p} u_{p y}\left(1-\frac{u_{e x}}{u_{p x}}\right) B \\
& -e q f_{h} u_{h y}\left(1-\frac{u_{e x}}{u_{h x}}\right) B .
\end{aligned}
$$

Adding Eqs. (24) and (25) we have

$$
\begin{gathered}
f_{p} \frac{d}{d x}\left(\frac{m_{p} u_{p x}^{2}}{2}+\frac{m_{p} u_{p y}^{2}}{2}\right)+f_{h} \frac{d}{d x}\left(\frac{m_{h} u_{h x}^{2}}{2}+\frac{m_{h} u_{h y}^{2}}{2}\right) \\
+u_{e x} \frac{d}{d x}\left[\frac{B^{2}}{2 \mu_{o}}+p_{e}\right]+u_{p x} \frac{d p_{p}}{d x}+u_{h x} \frac{d p_{h}}{d x}=0 .
\end{gathered}
$$

Using $u_{e x}=\left(u_{e o} B_{o}\right) / B$ and $p_{i}=p_{i o} \frac{n_{i}^{2}}{n_{i o}^{2}}$, the equation for the energy flux may be immediately integrated and written in the normalized form as:

$$
\begin{gathered}
\left(u_{p x}^{2}-1\right)+\alpha \mu\left(u_{h x}^{2}-1\right)+u_{p y}^{2}+\alpha \mu u_{h y}^{2}+\frac{2}{M_{f}^{2}}\left(\frac{1}{u_{e x}}-1\right) \\
+\frac{2}{M_{p}^{2}}\left(\frac{1}{u_{p x}}-1\right)+\frac{2 \alpha \mu}{M_{h}^{2}}\left(\frac{1}{u_{h x}}-1\right)=0 .
\end{gathered}
$$

This equation is readily interpreted. The first four terms are the changes in the kinetic energy fluxes of the protons and heavy ions. The term with the magnetic field $\left(2 / M_{A}^{2}\left(1 / u_{e x}-1\right)\right.$, where $\left.M_{A}=u_{o} / V_{A p}\right)$ is the Poynting 
flux, while the remaining three terms are fluxes of electron and ion enthalpies. Thus, the five variables, $u_{p x}, u_{h x}, u_{e x}$, $u_{p y}, u_{h y}$ of the system are governed by three conservation equations, namely (15), (17), (27), and Eq. (21), which provides an additional constraint on the velocities on the constituents (and charge neutrality $n_{e}=n_{p}+n_{h}$ ) and any of the differential equations of motion. For example, the term on the right-hand side of Eq. (19) may be determined in algebraic form (rather than differential form from Eq. (20)) by using conservation of energy flux:

$$
\begin{gathered}
\frac{q\left(u_{p y}-u_{h y}\right)}{u_{e x}}=-\frac{(1+\alpha \mu)}{u_{e x}} u_{h y}= \\
\mp \frac{q}{u_{e x}} \sqrt{\frac{(1+\alpha \mu)}{\alpha \mu}} \sqrt{E},
\end{gathered}
$$

where

$$
\begin{aligned}
E= & \left(1-u_{p x}^{2}\right)+\alpha \mu\left(1-u_{h x}^{2}\right)+\frac{2}{M_{f}^{2}}\left(1-\frac{1}{u_{e x}}\right) \\
& +\frac{2}{M_{p}^{2}}\left(1-\frac{1}{u_{p x}}\right)+\frac{2 \alpha \mu}{M_{h}^{2}}\left(1-\frac{1}{u_{h x}}\right)
\end{aligned}
$$

is the change of the total energy flux minus the term describing the kinetic energy flux of the transverse deflections of the protons and heavy ions. The problem therefore reduces to the following first order differential equation for $u_{p x}$

$\frac{d u_{p x}}{d x}= \pm q \sqrt{E} \sqrt{\frac{(1+\alpha \mu)}{\alpha \mu}} \frac{u_{p x}^{2}}{u_{e x}} \frac{N}{D}$,

where $N=(1+\alpha q) \alpha \mu D_{h}-\alpha q D_{e}$ and $D=\mu D_{p} D_{h}(1+$ $\alpha q)\left(\alpha q u_{p x}+u_{h x}\right)-\alpha q^{2} D_{p} D_{e} u_{p x}-\mu D_{h} D_{e} u_{h x}$. Here $D_{p}=u_{p x}^{2}\left(1-1 /\left(M_{p}^{2} u_{p x}^{3}\right)\right), D_{h}=u_{h x}^{2}\left(1-1 /\left(M_{h}^{2} u_{h x}^{3}\right)\right)$, $D_{e}=1 /\left(M_{f}^{2} u_{e x}\right)$. This equation (together with similar equations for $d u_{h x} / d x$ and $d u_{e x} / d x$ ) completely determines the properties and structure of stationary wave patterns in biion plasma with finite ion and electron temperatures. Hereafter we refer to it as the structure equation of the system in the same way as the equation governing the transition in a shock wave is called the shock structure equation.

\section{Nonlinear waves and solitons}

It has been shown recently by McKenzie et al. (2001a) that many features of stationary flows in a cold bi-ion plasma can be elucidated only by making use of the conservation of total momentum, quasi-charge neutrality and zero current in the $x$ direction. This combination of relations, called "the momentum hodograph", provides algebraic relations between the ion and electron speeds in the direction of unperturbed flow at any point in the structure. Although the inclusion of plasma pressure complicates this relation (now a quartic equation), it is useful to consider the changes in the shape of the momentum hodograph arising from finite temperature.
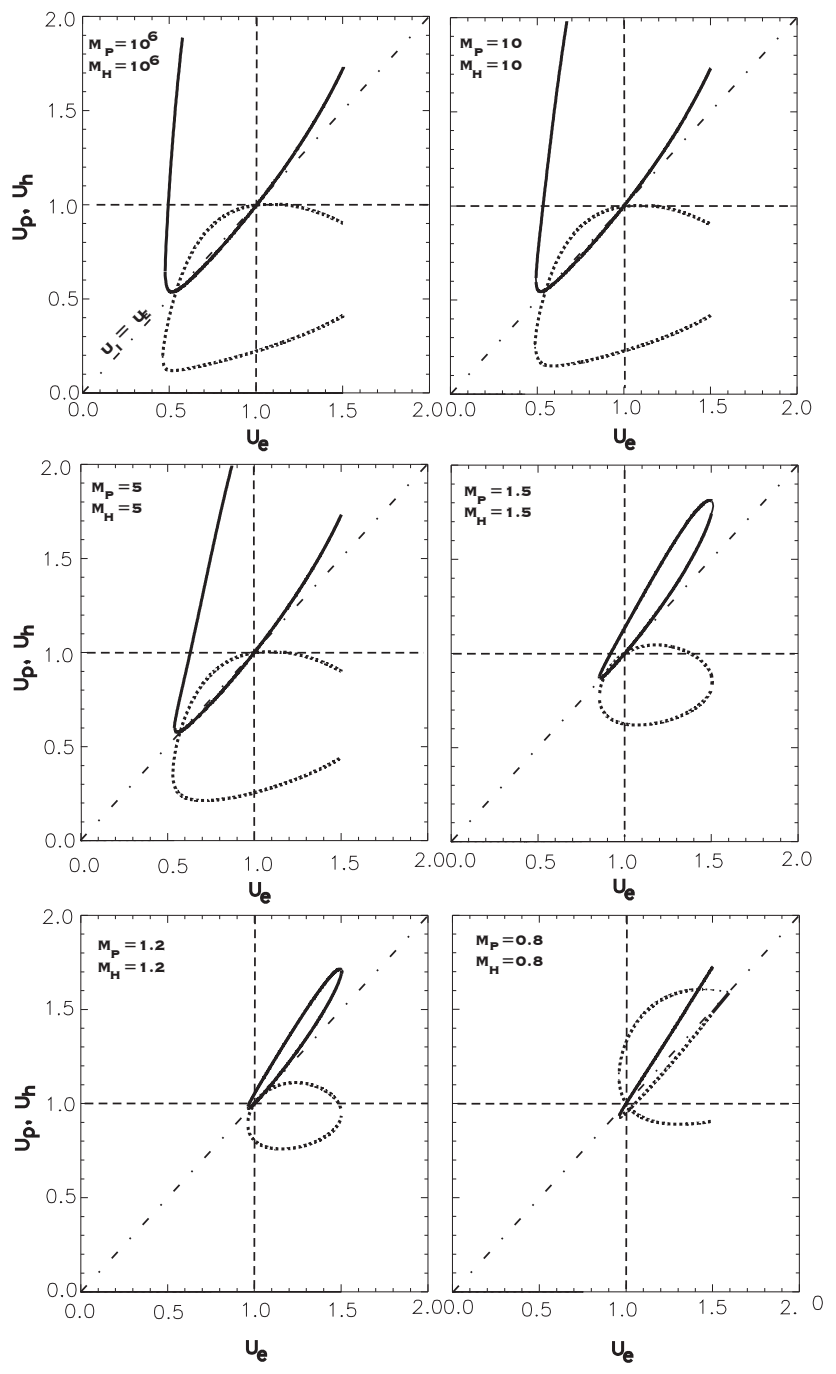

Fig. 1. Momentum hodographs for different ion-sonic Mach numbers $\left(M_{p}, M_{h}\right)$ and $\alpha=0.2, \mu=15, \beta_{e}=0, M_{A}=0.8$. Solid (dotted) curves correspond to the proton (heavy ion) speed along the direction of unperturbed flow. Compressive solutions evolve along the curves to the left of the initial point $\left(u_{e}<1\right)$, whereas rarefaction solutions evolve along the hodograph curves to the right. The dashed-dotted line is shown to indicate a differential streaming of the ion species with respect to the electron speed.

Figure 1 shows the locii of $u_{p x}$ (solid line) and $u_{h x}$ (dotted lines) as a function of $u_{e x}$ for different sets of parameters $M_{f}, M_{p}, M_{h}, \alpha, \mu$. The top left panel shows the momentum hodograph for the parameters used in the cold ion case $\left(M_{p}=M_{h}=\infty, M_{f}=M_{A}\right.$, where $M_{A}$ is the Alfvénic Mach number based on the proton mass density) for which a soliton solution was given in Sauer et al. (2000) and McKenzie et al. (2001a). Note that the inclusion of electron pressure only modifies the Alfvén Mach number to the fast Mach number $M_{f}$ without introducing any qualitative change in the structure equation and therefore, for simplicity, we may consider the electrons as cold. All solutions of the structure equation evolve from the initial point 
$\left(u_{p x}=u_{h x}=u_{e x}=1\right)$. Compressive $(B>1)$ solutions evolve to the left $\left(u_{e x}<1\right)$, whereas rarefaction $(B<1)$ solutions evolve along the curves to the right. The momentum hodograph shown in the top left panel is very nearly the same as the one for the cold case. In compressive solutions the protons and heavy ions are decelerated along with the electrons. Since all species move with different speeds (the heavy ions run ahead of, and the protons lag behind, the electrons), a strong coupling caused by the Lorentz force (constrained by the quasi-charge neutrality) ensures the existence of the soliton structure. At $u_{e x} \sim 0.5$, all fluids are decelerated to the same speed, after which point the protons, by taking momentum from the heavy ions, are sharply accelerated, while the heavy ions now lag behind the electrons. At some "equilibrium" point (given by the zero of the numerator of Eq. 30), the system evolves back along the hodograph to the initial point by reflection about this equilibrium point.

However, the momentum hodograph alone does not tell us where this reflection or equilibrium point is located. This is determined by analyzing the structure of Eq. (30). Before discussing the possible structures embedded in this equation, we briefly consider how finite pressure affects the momentum hodograph. A decrease in the sound Mach numbers (increase of pressure) shifts the point where all fluids stream with the same speed towards the initial point and therefore the maximum possible compression strength decreases. At $M_{p}=M_{h}=1.2$, the protons, near the initial point, immediately begin to run ahead of the heavy ions and electrons, with the result that the point where all fluids move with the same velocity disappears altogether from the compressive part of the hodograph and reappears in the rarefaction branch. Note that in this case, a compressive soliton solution cannot be constructed because, from the transverse equation of motion,

$u_{p x} \frac{d u_{p y}}{d x}=e\left(u_{e x}-u_{p x}\right) B$

it follows that since the deflection in the $y$-direction must be an odd function of $x$, a point where $u_{e x}=u_{p x}$ must be reached before the centre of the structure. In the "subsonic" regime $\left(M_{p}<1 M_{h}<1\right)$, an intersection between line $u_{p x}\left(u_{h x}\right)=u_{e x}$ with the hodograph curves occurs for $u_{e x}>1$. In this rarefaction branch the heavy ions (protons) are decelerated (accelerated). However, as we will see below, the structure of the solutions is strongly affected by the position of critical sonic points (where $d u_{i x} / d x \rightarrow \infty$ ) with the result that rarefaction solitons cannot be constructed.

Figure 2 shows the phase curves in the planes $\left(d u_{p x} / d x\right.$, $\left.u_{p x}\right),\left(d u_{h x} / d x, u_{h x}\right)$ for the same set of parameters $M_{A}, M_{p}, M_{h}, \alpha, \mu$ for the momentum hodographs shown in Fig. 1. At very large ion sound Mach numbers we have a soliton solution similar to the cold case. The equilibrium point from which the system evolves back to the initial state is reached at $u_{p x} \sim 0.6$. Compression of the protons is followed by a sudden rarefaction and acceleration, while the heavy ions continue to be decelerated. It can also be observed that a smooth rarefaction soliton cannot exist because a point $d u_{i x} / d x \rightarrow \infty$ is reached before the expansion is completed (quite apart from the constraints related to transverse deflections). At $M_{p}=M_{h}=10$, the equilibrium point is reached at larger values of $u_{p x}(\sim 0.78)$, which implies that a rarefaction substructure embedded in the soliton is strengthened. At $M_{p}=M_{h}=8$, the proton velocity at first decreases by a factor of two and then the protons are reaccelerated almost up to the initial speed in the centre of the structure, while the heavy ions continue be decelerated down to $(\sim 0.17)$. A further increase in the ion pressure results in the disappearance of a smooth soliton because a critical point for the protons is reached after the end of a compression phase and their reacceleration up to $\sim 0.62\left(\right.$ see $\left.M_{p}=M_{h}=5\right)$.

It is interesting to note that at smaller Mach numbers, soliton solutions again reappear $\left(M_{p}=M_{h}=1.5\right)$. The significant difference in this case is that a soliton comprises only a compression structure. The rarefaction part is detached from it and forms another solution which can only be reached through a discontinuity. However, this solution is not a periodical because of critical points at $u_{p x} \sim 1.15$ and $u_{h x} \sim 0.62$. An example of periodical solution (the phase trajectories for the case $M_{A}=1, M_{i}=20$ ) is shown in the right bottom panel of Fig. 2. Here the protons and heavy ions are executing an oscillating motion, not by a smooth transition from the initial point, but only through a jump (discontinuity)

Figure 3 shows examples of numerical solutions of the structure equation for both solitons and periodical cnoidal waves.

\section{The Effect of plasma pressure on soliton structure}

We now examine the structure equation in more detail to obtain a clearer understanding of the different possible types of stationary solutions. The integral curves of this rather complicated differential Eq. (30) can be constructed from our knowledge of the behaviour of the right-hand side (RHS). The zeroes of this expression determine the location of the equilibrium points (where $d u_{p x} / d x=0$ ), which correspond to the amplitude of the wave. The RHS has zeroes at the points where $E\left(u_{p x}\right)=0$ or at the points where

$N=(1+\alpha q) \mu D_{h}-q D_{e}=0$.

Figure 4a shows $E\left(u_{p x}\right)$ (dashed curves)and $N\left(u_{p x}\right)$ (dotted curves) as a function of $u_{p x}$ for several sets of the parameters used in Figs. 1 and 2 . The zeroes of $E\left(u_{p x}\right)$ and $N\left(u_{p x}\right)$ in a compressive part $\left(u_{p x}<1\right)$ correspond to the amplitudes of possible structures. One of the zeroes $(N=0)$ determines the maximum compression in the structure. Another one $(E=0)$ gives the amplitude of the solution in the centre (an equilibrium point). Figure $4 \mathrm{~b}$ shows the behaviour of the denominator of the structure equation

$$
\begin{aligned}
D= & \mu(1+\alpha q) D_{p} D_{h}\left(u_{h x}+\alpha q u_{p x}\right)-\alpha q^{2} D_{p} D_{e} u_{p x} \\
& -\mu D_{h} D_{e} u_{h} .
\end{aligned}
$$

This tells us the location of critical points where $\frac{d u_{i x}}{d x} \rightarrow \infty$. Only a combined analysis of the behaviour of both $E\left(u_{p x}\right)$ 

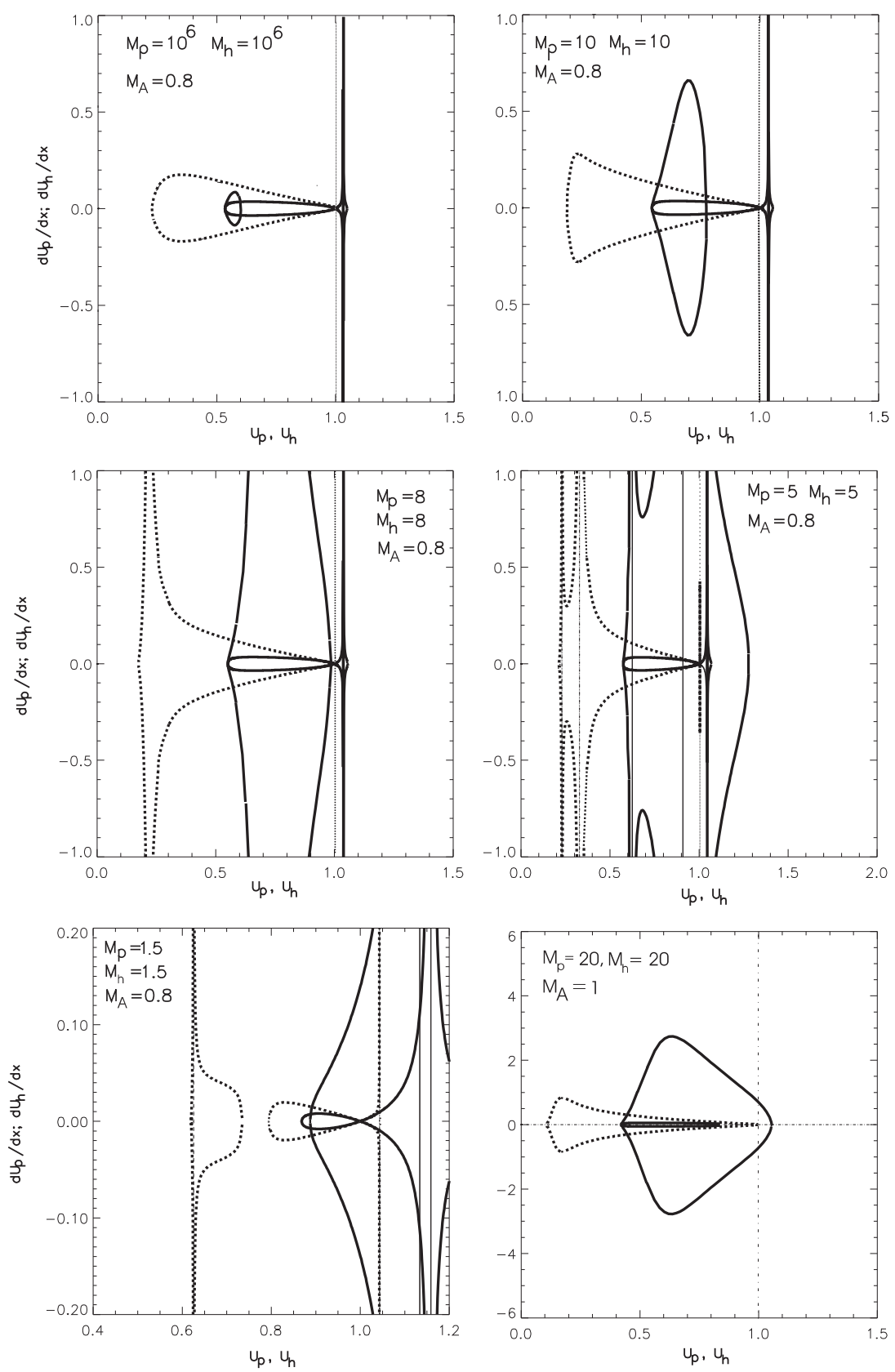

Fig. 2. Phase curves in the planes $\left(d u_{p x} / d x, u_{p x}\right),\left(d u_{h x} / d x, u_{h x}\right)$ for the same set of parameters $M_{A}, M_{p}, M_{h}, \alpha, \mu$ as for the momentum hodographs shown in Fig. 1. The left top panel corresponds to the case of cold ions. Arrows indicate the variations in $u_{p x}$ and $u_{h x}$ for the compressive solution. Rarefaction solutions do not exist because $d u_{i x} / d x \rightarrow \infty$ before the expansion is completed. At the Mach numbers $\sim 1.5<M_{p}, M_{h}<\sim 7.5$, smooth compressive solitons also cannot be constructed because critical points are reached before the proton expansion in rarefaction cores is complete. At $M_{p}=M_{h}=1.5$, the rarefaction core is detached from the compressive part and the soliton structure is changed. The right bottom panel shows an example of phase curves typical for periodical nonlinear waves. The protons and the heavy ions are moving in an oscillating manner.

and $D\left(u_{p x}\right)$ provides sufficient information to determine the nature of the structure.

It is important to note that soliton-like solutions exist only for $E \geq 0$. At large ion Mach numbers in the vicinity of $u_{p x}=1, E$ is positive for $u_{p x}<1$ and for $u_{p x}>1$, and a priori, one could expect the existence of rarefaction solitons. However critical points, where $d u_{p x} / d x=\infty$, may inter- vene before equilibrium points are reached. It is worth noting that at large $M_{i}(=10)$, the energy flux integral $E\left(u_{p x}\right)$ evolves to the left from the initial point "O" $\left(u_{p x}=1\right)$, and increases for compressive type of solutions, reaches a maximum, and returns to zero along another path. This point determines the amplitude of the soliton at its centre. At the same time, the denominator function $D\left(u_{p x}\right)$ evolves in the 


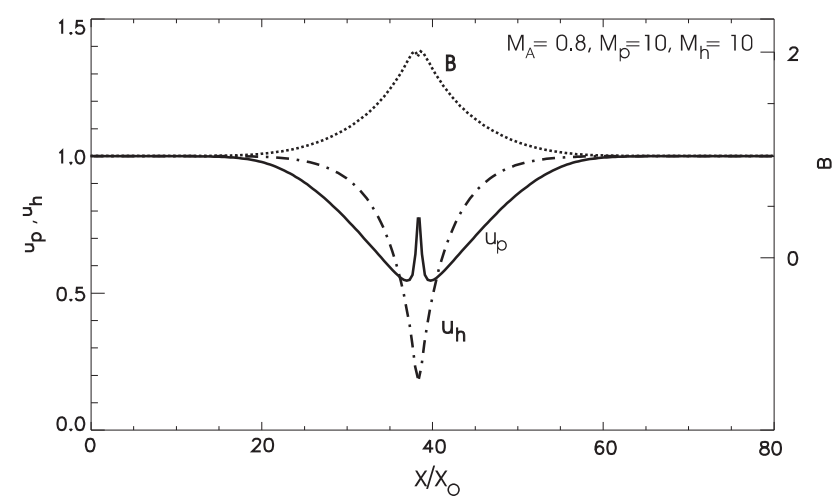

a)
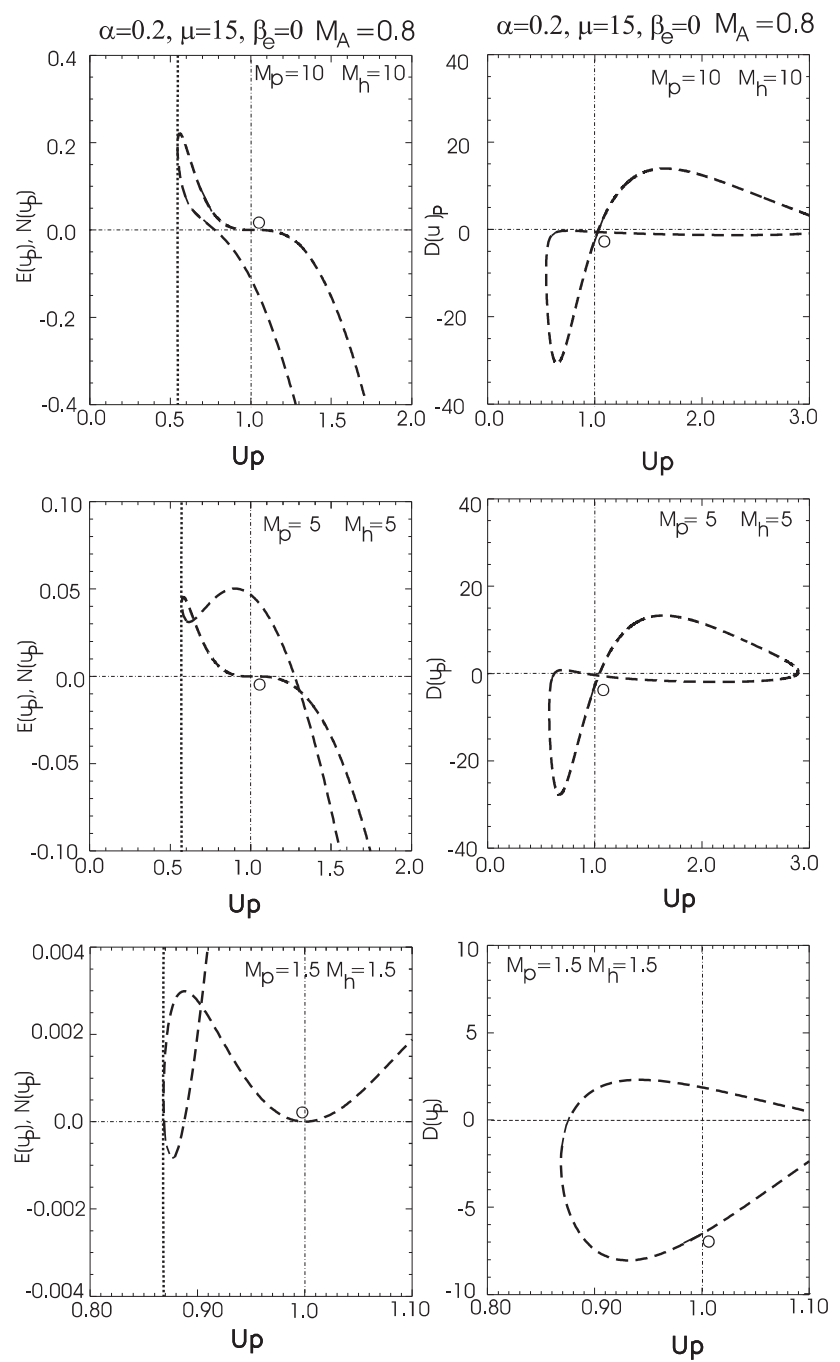

Fig. 4. (a) The energy flux integral $E\left(u_{p x}\right)$ (dashed curves) and the function $N\left(u_{p x}\right)$ (dotted curves) as functions of $u_{p x}$. The zeroes of these functions determine the location of the equilibrium points where $d u_{p x} / d x=0$. (b) The denominator $D\left(u_{p x}\right)$ of the structure equation which determines the location of the critical point where $d u_{i x} / d x=\infty$, as a function of $u_{p x}$.

A further increase in ion pressure brings about a local minimum in the curve of $E\left(u_{p x}\right)$ which could evolve to equilibrium points with $u_{p x}>1$. However, these equilibrium points are not realized because the zeroes of $D\left(u_{p x}\right)$ intervene. For example, at $M_{p}=M_{h}=5$, two zeroes of $D$ occur in the compressional branch before the equilibrium point $\left(u_{p x}=\sim 1.3\right)$, therefore taking out the construction of a smooth soliton. An interesting feature appears when $M_{p}=$ $M_{h}=1.5$. The value of the local minimum of $E\left(u_{p x}\right)$ decreases with decreasing $M_{i}$ and reaches zero, and at $M_{p}=$ $M_{h}=1.5$, it becomes negative. In this case "a new" equilibrium point arises, already in the compressive stage of the proton flow, and is reached just before a reflection point. Thus, a soliton can again be constructed although its structure no

compressional part from a point "O" without going through zero. For rarefaction solutions, $D\left(u_{p x}\right)$ moves to the right and goes through zero before $E\left(u_{p x}\right)$ reaches its zero. Therefore, smooth rarefaction solutions cannot be constructed. 
longer contains a rarefaction core. At smaller Mach numbers, $E\left(u_{p x}\right)$ is negative in the neighbourhood of $u_{p x}=1$ so that solitons cannot be constructed. For "subsonic" Mach numbers, where the momentum hodographs may permit rarefaction $\left(u_{e x}>1\right)$ solitons, a reflection point $(D=0)$ is always reached before an equilibrium point $(E=0)$.

The solution of the system of Eqs. (15), (29) and (21), which represent momentum conservation, energy flux conservation (minus the terms describing the kinetic energy flux of transverse deflection) and the constraint on the current $j_{x}$, yields the amplitudes $\left(u_{p x}, u_{h x}, B=1 / u_{e x}\right)$ of stationary solutions for given values of Mach numbers (transverse speeds of ion species become zeroes at the center of stationary structures). These relations $u_{p x} / u_{o}, u_{h x} / u_{o}, B / B_{o}$ may be thought of as the analogue of the Rankine-Hugoniot relations for shocks. However, two important points must be noted. The energy flux associated with the transverse components of the ion velocities is not included in the equation $E=0$. This, however, is not a severe limitation because

$u_{p x}=\int \frac{e}{m_{p}} \frac{u_{e x}-u_{p x}}{u_{p x}} B d x$

and for a "shock" transition with a thickness $\Delta \rightarrow 0$, the downstream value of $u_{p y} \rightarrow 0$ (Motschmann et al., 1991). The second important difference from standard RankineHugoniot relations is that we only consider adiabatic flows. Even in the case of cold species upstream of a "shock" transition, one must take into account finite pressures of species downstream of the transition. Nevertheless, for weak shocks in a bi-ion plasma with small thermal pressures, as compared to the magnetic pressure, the analogy seems reasonable.

The solid thick black curves in Figs. 5 and 6 represent solutions of the system of Eqs. (15), (21), (29) for different sets of Mach numbers. Figure 5 shows $u_{p x} / u_{o}, u_{h x} / u_{o}$, $B / B_{o}$ as a function of $M_{i}\left(=M_{p}=M_{h}\right)$ for $M_{A}=0.8$. The thin solid black curves depict solutions of the same system of equations where, instead of equation $E=0$, we take Eq. (32) as determining the maximum value of the proton compression $\left(N\left(u_{p x}\right)=0\right)$. The red curves show the amplitudes at which reflection points (where $d u_{i x} / d x \rightarrow \infty$ ) are reached (the system of equations solved are Eqs. (15), (21) and the equation $D=0$, where $D$ is the denominator (33) of the structure equation). The solutions $(C)$ and $\left(C_{m}\right)$ correspond to compressive flows, whereas solution $(R)$ corresponds to a rarefaction flows. The range in which soliton solutions exist are readily recognized from the blue lines $u_{p x} / u_{o}=1$. Note that the transition $C \longleftrightarrow \rightarrow C_{m}$ can be fulfilled without crossing critical points (red lines) only for $M_{i}>\sim 7.5$. Rarefaction solutions (for the protons) cannot be constructed at any $M_{i}$ because critical points are reached before the final expansion is completed. An interesting feature is observed at $\sim 1.3 \leq M_{i} \leq \sim 1.5$ where a bifurcation of the curves appears, giving rise to a new, additional solution. This occurs because a new equilibrium point arises where $E\left(u_{p x}\right)=0$ and $d E / d u_{p x}=0$ simultaneously, and the rarefaction core is disconnected from the soliton structure. At smaller values
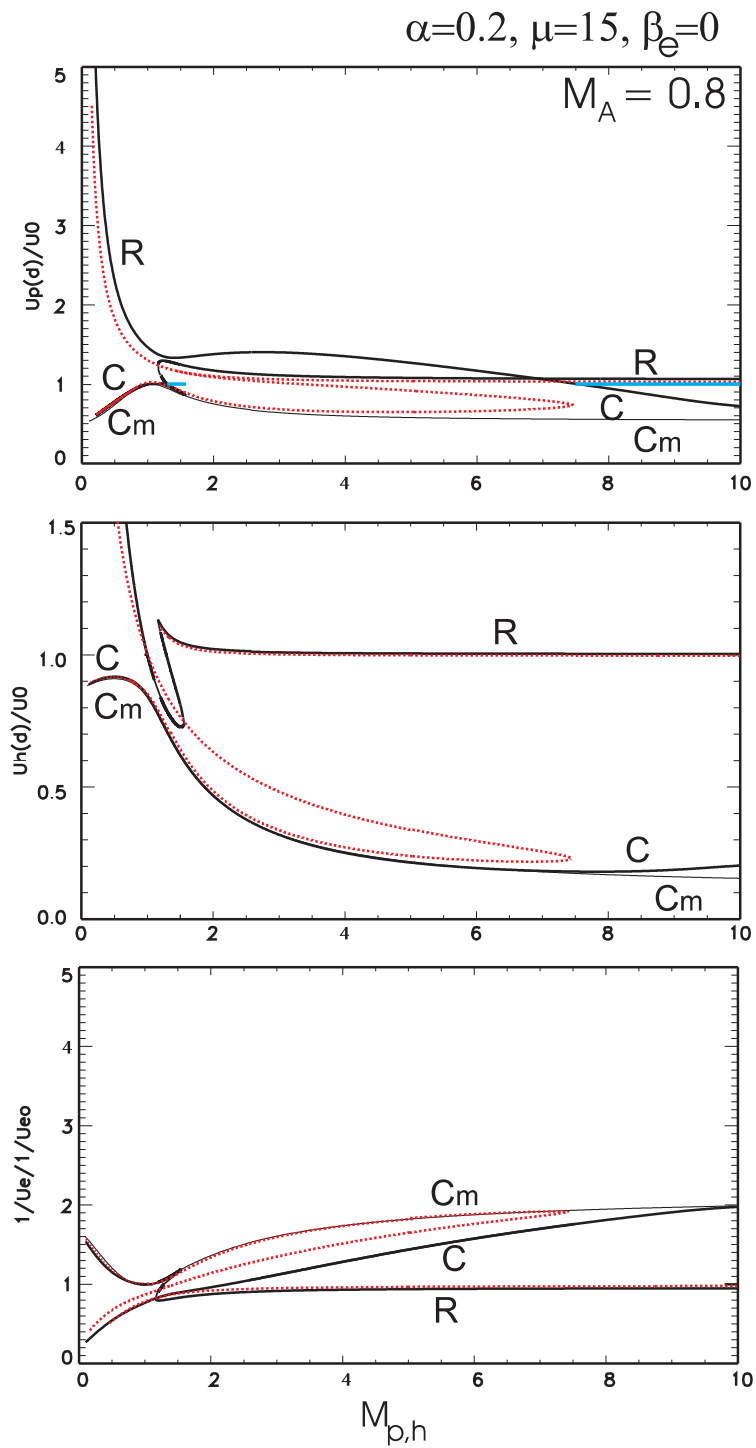

Fig. 5. Compression ratios $u_{p x} / u_{o}, u_{h x} / u_{o}, B / B_{o}$ of stationary solutions, for Alfvén Mach number $M_{A}=0.8$, as a function of ion-sonic Mach numbers $\left(M_{p}=M_{h}\right)$. Solid black curves represent the amplitudes of the solutions at the centre of the structures. Thin black curves depict the maximum values of the compression ratios. Red lines correspond to the values at which reflection points are reached. The range of $M_{p, h}$, in which solitons exist, are shown by blue lines at $u_{p x} / u_{o}=1$.

of $M_{i}$, reflection points are reached before equilibrium points are attained.

Figure 6 shows the amplitudes of the proton flow speeds as a function of the Alfvén Mach number for given values of ion pressures. At $M_{i}=10$, compressive solitons exist only for $M_{A}<\sim 0.85$. In the range $\sim 0.85 \geq M_{A} \geq \sim 0.75$, solitons contain an embedded rarefaction core. At smaller values of $M_{A}$ (to the left from the minimum in the curve $C$ ), only the compressive part remains (the curves $C$ and $C_{m}$ almost merge although the curve $C_{m}$ remains outside). At $0.4<M_{A}<0.5$, only rarefactions (not smooth) solutions 

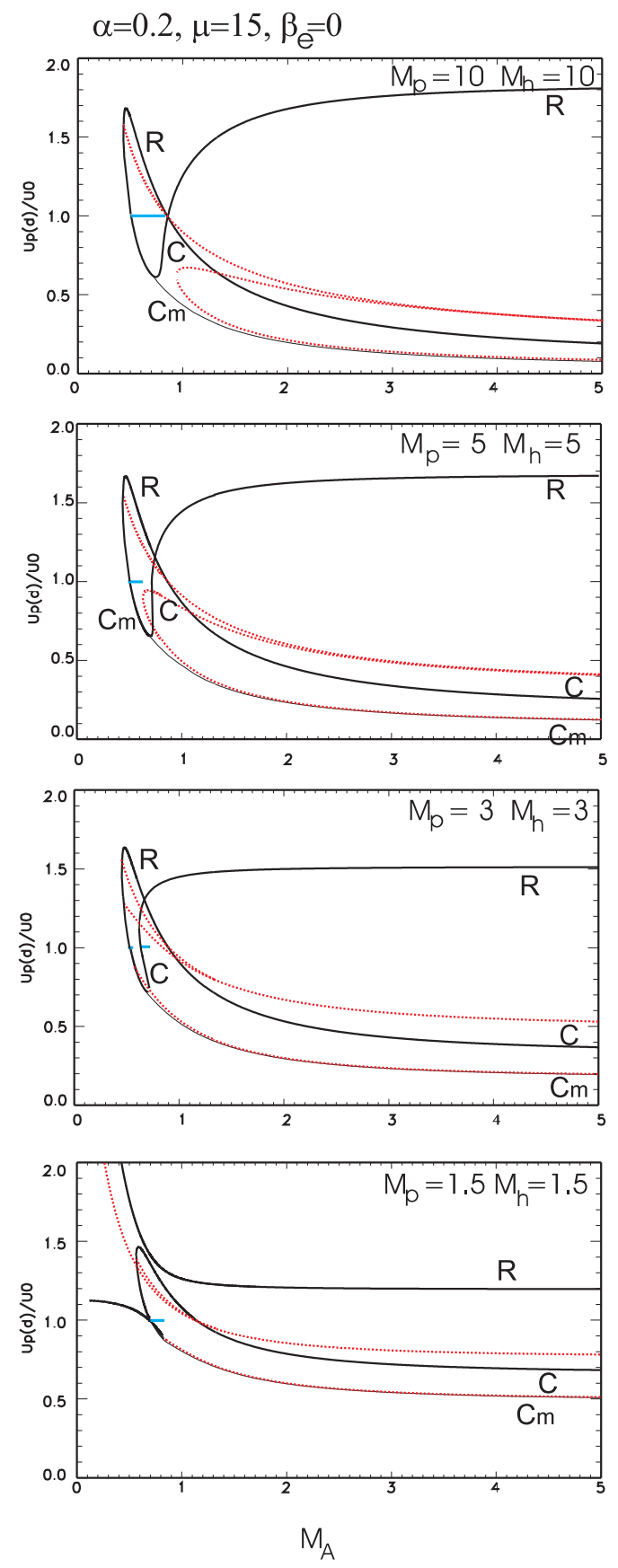

Fig. 6. Compression ratios $u_{p x} / u_{o}$ of stationary solutions for the set of ion-sonic Mach numbers $\left(M_{p}=M_{h}\right)$ as a function of Alfvén Mach number $M_{A}$.

exist. At $M_{A}<\sim 0.4$, there are no solitons since $E\left(u_{p x}\right)$ becomes negative for all $u_{p x}$. At $M_{i}=5$, the gap for solitons becomes smaller $\left(\sim 0.5 \leq M_{A} \leq \sim 0.6\right)$ and only compressive solutions are possible (to the left of the point where the curves $C$ and $C_{m}$ merge). There is also a very narrow bifurcation region at $M_{A} \sim 0.75$ where a new equilibrium point appears and a compressive soliton may exist. This bifurcation becomes clearer at $M_{i}=3$ and $M_{A} \sim 0.65$. Another region of solitons for $M_{i}=3$ occurs where $M_{A} \sim 0.55$. Both regions are separated by a gap in which $E\left(u_{p x}\right)$ is negative in the vicinity of the initial point and critical points prevent even the existence of periodical solutions. At $M_{i}=1.5 \mathrm{com}-$ pressive solitons of small amplitude exist only in a region of bifurcation $\left(M_{A} \sim 0.7\right)$. Although at $M_{i}=1.5$, solutions exist even at very small $M_{A}$, neither smooth solitons or periodical waves can be constructed.

\section{Critical Mach numbers}

The set of results presented above for specific combinations of Mach numbers, demonstrates the complicated behaviour of the energy flux integral $E\left(u_{p x}\right)$ and denominator of the structure equation $D\left(u_{p x}\right)$ whose zeros restrict the possible trajectories of the integral curves by "choking the flow". The sample of results can be placed in a more general context in the following way. A necessary condition for the existence of solitons is the condition that the energy flux integral $E\left(u_{p x}\right)$ must be positive in the neighbourhood of the initial point. To examine the behaviour of $E\left(u_{p x}\right)$ note that

$$
\begin{aligned}
& \frac{d E\left(u_{p x}\right)}{d x}=2\left(\frac{1}{M_{p}^{2} u_{p x}^{3}}-1\right)\left(u_{p x}-u_{e x}\right) \\
& \quad+2 \alpha \mu\left(\frac{1}{M_{h}^{2} u_{h x}^{3}}-1\right)\left(u_{h x}-u_{e x}\right) \frac{d u_{h x}}{d u_{p x}}
\end{aligned}
$$

which shows that $E\left(u_{p x}\right)$ and $d E\left(u_{p x}\right) / d x$ go to zero at the initial point $u_{i x}=1$. Note also that at the "charge" neutral point, where all fluids move at the same speed $\left(u_{p x}=\right.$ $\left.u_{e x}=u_{h x}\right)$ the energy function $E\left(u_{p x}\right)$ has a local maximum. Therefore, in the neighbourhood of the initial point, $E\left(u_{p x}\right)$ behaves as

$E\left(u_{p x}\right) \approx a_{2} \delta^{2}$

in which

$$
\begin{aligned}
& a_{2}=\frac{2(1-S)}{1+\alpha q}\left[\left(\frac{1}{M_{p}^{2}}-1\right) \alpha q-\alpha \mu\left(\frac{1}{M_{h}^{2}}-1\right) S\right], \\
& S=-\frac{\left(1 / M_{p}^{2}-1\right)(1+\alpha q)+1 / M_{f}^{2}}{\alpha \mu\left(1 / M_{h}^{2}-1\right)(1+\alpha q)+\alpha q / M_{f}^{2}} .
\end{aligned}
$$

Hence, $E$ is positive if

$$
1-S>0, \quad \text { and }\left(\frac{1}{M_{p}^{2}}-1\right) q-\left(\frac{1}{M_{h}^{2}}-1\right) \mu S>0,
$$

or

$$
1-S<0, \quad \text { and }\left(\frac{1}{M_{p}^{2}}-1\right) q-\left(\frac{1}{M_{h}^{2}}-1\right) \mu S<0 .
$$

These conditions determine the range of the Mach numbers in which soliton solutions are possible. In the case of cold protons and heavy ions $\left(M_{p} \rightarrow \infty, M_{h} \rightarrow \infty\right)$ the conditions reduce to

$$
M_{f}^{2}>\frac{1}{1+\alpha \mu}, \quad \text { and } M_{f}^{2}<\frac{\alpha q^{2}+\mu}{\mu(1+\alpha q)^{2}}
$$



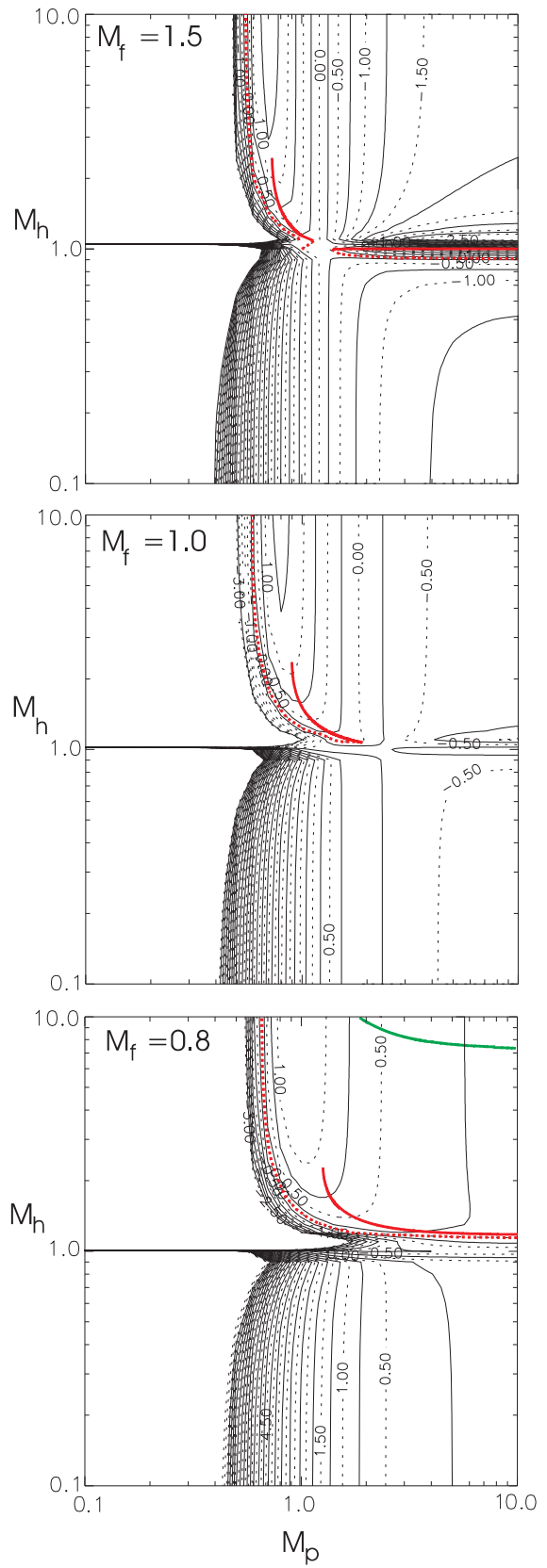

Fig. 7. Two-dimensional contour plots of the coefficient $a_{2}$ as a function of $M_{p}$ and $M_{h}$ for super-Alfvénic $\left(M_{A}=1.5\right)$, Alfvénic $\left(M_{A}=1\right)$ and sub-Alfvénic $\left(M_{A}=0.8\right)$ flows. The plots encompass the range of the function $a_{2}$ from -3 up to +12 with an interval 0.25 between the contour lines. Solid green curves provide the locii of critical Mach numbers. Above this curve smooth soliton solutions can be constructed. The solid red curves provide another set of the upper critical Mach numbers. Solitons exist also in the regions bounded by solid and dotted red lines.

or if $\alpha q^{2} \ll \mu$, to

$M_{f}^{2}>\frac{1}{1+\alpha \mu}, \quad$ and $M_{f}^{2}<\frac{1}{(1+\alpha q)^{2}}$.

A second possible range of Mach numbers where solitons may exist (condition 39) is fulfilled only for a very lim-

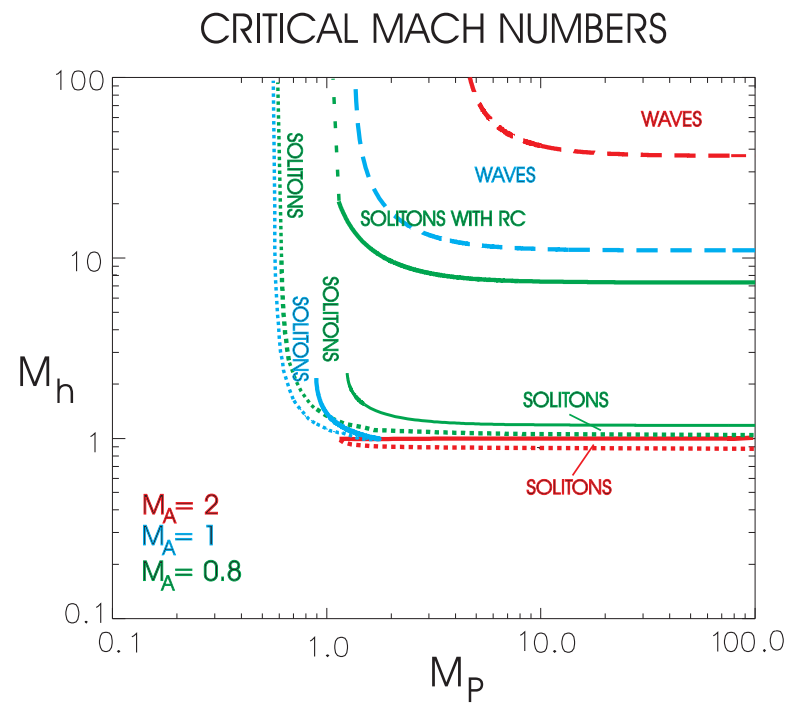

Fig. 8. The range in the ion-sonic Mach numbers $M_{p}, M_{h}$ where solitons or stationary nonlinear waves may exist. Solid (dashed) lines are locii of critical Mach numbers for smooth solitons (periodical waves). Dotted lines depict $E\left(u_{p}\right)=0$.

ited range of parameters $\alpha$ and $\mu$ (if $\mu\left(1+\alpha q^{2}\right)^{2}>(1+$ $\left.\alpha \mu)\left(\alpha q^{2}+\mu\right)\right)$. In the cold case, the lower limit of Mach number in (41) $M_{f}\left(M_{f}^{2}=1 /(1+\alpha \mu)\right)$ is essentially the Alfvén speed based on the total ion mass density, and the upper limit is the Alfvén speed reduced by the factor $(1+\alpha q)$. For finite ion temperature, the condition $1-S>0$ can also be interpreted as

$M_{f_{b i}}>1$,

where we may define the fast Mach number for a bi-ion plasma as

$\frac{1}{M_{f_{b i}}^{2}}=\frac{1}{1+\alpha \mu}\left(\frac{1}{M_{p}^{2}}+\frac{\alpha \mu}{M_{h}^{2}}+\frac{1}{M_{f}^{2}}\right)$.

Figure 7 shows the two-dimensional contour plots of the function (37) $a_{2}$ as a function of $M_{p}$ and $M_{h}$ for superAlfvénic $\left(M_{A}=1.5\right)$, Alfvénic $\left(M_{A}=1\right)$, and sub-Alfvénic $\left(M_{A}=0.8\right)$ flows. Soliton solutions can be found only where this function (and consequently the energy flux function) is positive. In the super-Alfvénic case, solitons may exist in 3 regions on the plane $\left(M_{p}-M_{h}\right)$ (the cut of the three-dimensional $M_{A}-M_{p}-M_{h}$ parameter space): a) $\left.0.9 \leq M_{p} \leq 1.2, M_{h} \geq \sim 1.2 ; b\right) M_{p} \geq 1.5,0.9 \leq$ $M_{h} \leq \sim 1$; c) $0.5 \leq 0.5 \leq M_{p} \leq 1, M_{h} \leq 1.05$. For subAlfvénic flow, the first region expands to high $M_{p}$ values and the second and the third regions are merged. Consequently, the parameter space in the $M_{p}-M_{h}$ plane in which solitons are permitted is greatly expanded.

However, in many cases, smooth soliton solutions cannot be constructed because critical points intervene. Therefore, it is useful to examine critical Mach numbers and critical amplitudes of solitons. These values are determined by the condition that a local maximum of $D\left(u_{p x}\right)$ becomes zero so that 
$D\left(u_{p x}\right)$ possesses a double root. The solution of the system of equations $D\left(u_{p x}\right)=0, d D\left(u_{p x}\right) / d x=0$, the equation of the momentum conservation (15) and Eq. (21) $\left(j_{x}=0\right)$ for given values of one of the Mach numbers ( $\alpha$ and $\mu$ are also fixed) yields the range of the two remaining critical Mach numbers in which smooth solitons or periodical waves are possible. The green curves on the contour plots shown in Fig. 6, depict such critical Mach numbers. At Mach numbers $M_{A}=0.8$ and $M_{p}, M_{h}$ above this critical curve, smooth soliton solutions exist.

By examining the structure equation for Mach numbers varying in the plane $\left(M_{p}-M_{h}\right)$, we have found that soliton solutions also exist at small $M_{i}$. A second set of critical Mach numbers can be determined by the condition that a local minimum of the energy flux integral $E\left(u_{p x}\right)$ goes to zero. In this case the system of equations comprises equations $E\left(u_{p x}\right)=0$ and $d E\left(u_{p x}\right) / d x=0$, together with those determining the momentum hodograph. Below these critical Mach numbers, compressive solutions, without a rarefaction core can be constructed in the range of parameters where $E\left(u_{p x}\right) \geq 0$. The solid red curves in Fig. 7 provide the upper critical Mach numbers. Dotted red curves show the locii of the Mach numbers where $E\left(u_{p x}\right)=0$. The range of Mach numbers $M_{p}-M_{h}$ between the solid and dotted curves gives the values of the proton and heavy ion velocities at which smooth compressive solitons can be constructed. It is interesting to note that at $M_{p}<0.9\left(M_{f}=1\right)$ and $M_{p}<1.3\left(M_{f}=0.8\right)$, in the regions where $E\left(u_{p}\right)>0$ (to the right of the dotted red curves), smooth compressive solitons also exist. There are no critical values of $M_{h}$ at these $M_{p}$ numbers. In "sub-heavy-sonic" $\left(M_{h}<1\right)$ and "sub-Alfvénic" $\left(M_{f}<1\right)$ regimes, smooth soliton solutions are not possible although at the super-Alfvénic speeds (e.g. $M_{f}=1.5$ in Fig. 7), compressive solitons at small amplitude $\left(u_{p} \sim u_{h} \sim 0.96\right)$ survive at $1>M_{h}>\sim 0.9$ and $M_{p} \geq \sim 1.3$.

Figure 8 shows the ranges of ion sound Mach numbers in the $M_{p}-M_{h}$ planes where solitons or stationary nonlinear waves may exist. Solid curves correspond to the locii of the critical Mach numbers for smooth solitons. It was noted that for solitons another additional constraint must be fulfilled, namely that $E\left(u_{p x}\right) \geq 0$ in the vicinity of the initial point. If $E\left(u_{p x}\right)$ is negative and critical points do not appear, another class of solutions (nonlinear stationary waves) can be found. The amplitudes of these waves also have critical values at certain critical Mach numbers. Dashed curves in Fig. 8 are locii of the critical values for periodical stationary waves. Dotted curves show where $E\left(u_{p}\right)=0$. Solitons or periodical nonlinear waves can be constructed at high Mach numbers (right top corners bounded by the critical curves). At $M_{A}=0.8$, solitons have a rarefaction core (RC). Regions bounded by the dashed curves $\left(M_{A}=1\right.$ and 2$)$ correspond to Mach numbers $M_{p}$ and $M_{h}$ at which periodical solutions exist. For super-Alfvénic flows $\left(M_{A}=2\right)$, compressive soliton solutions are possible only at $M_{p}>\sim 1$ and $1>M_{h}>\sim 0.9$ (the region between the solid and dotted red curves, see also Fig. 7 for the case $M_{A}=1.5$ ). At $M_{A}=1$, solitons may be found in the region bordered by the solid (a second set of the critical curves) and dotted blue curves. At $M_{A}=0.8$, soliton compressive solutions also exist at low heavy-sound Mach numbers $M_{h} \geq \sim 1$. The permitted region (between the solid and dotted green curves) gradually widens at $M_{p} \sim 1$ and expands to large heavy-sound Mach numbers $M_{h}$. Across the dashed green curve at $M_{h}>\sim 10$ there is a smooth transition between compressive solitons with and without a rarefaction core.

\section{Summary and conclusions}

We have investigated the structure of stationary nonlinear periodical waves and solitons propagating perpendicular to the magnetic field in a warm bi-ion plasma, using multi-fluid equations. It is well known that in transverse solitons propagating in a plasma consisting only of protons and electrons, finite ion inertia plays no role. Dispersion effects occur only at smaller scales where finite electron inertia progressively decouples the magnetic field from the fluid (see e.g. Adlam and Allen, 1958; Sagdeev, 1966; Tidman and Krall, 1971; McKenzie et al., 2001b). In a bi-ion plasma a new dispersion length appears. The dispersion equation for stationary waves follows from linearizing the structure Eq. (30) about the initial state where $u_{p x}=u_{h x}=u_{e}=1$, and seeking exponential solutions $\exp (\kappa x)$. This analysis is made more clear by noting that the behaviour of the right-hand-side (RHS) of Eq. (30) near the initial point, is mainly controlled by the energy flux function $E\left(u_{p x}, u_{h x}, u_{e}\right)$ (29) which has a double zero at $u_{i x}=1$. Other terms on the RHS can be expressed as $f\left(M_{p}, M_{h}, M_{f}, u_{i x}\right)$ at $u_{i x}=1$. A more accurate linearization procedure confirms this approach. $E\left(u_{i x}\right)$ must be expanded in powers of $u_{p x}-1=\delta$ up to $\delta^{2}$, so that the linearized Eq. (30) may be written as

$\frac{d\left(u_{p x}-1\right)}{d x}=\sqrt{a_{2}}\left(u_{p x}-1\right) f_{u_{i}=1}$,

and the exponent $\kappa$ is therefore

$\kappa= \pm \sqrt{a_{2}} f_{u_{i}=1}$.

Thus, evanescent solutions $\left(\kappa^{2}>0\right)$ (growing or decaying) are possible only if $a_{2}>0$, i.e. in accordance with the analysis made in Sect. 6. Propagating solutions $\exp (i \omega t-i k x)$ can be obtained from (46) by the transformations $\kappa \rightarrow-i k$ and $u_{o}=v_{p h} \equiv \omega / k$. The latter implies the transformations $M_{f} \rightarrow v_{p h} / v_{A p}, M_{p} \rightarrow v_{p h} / c_{p}, M_{h} \rightarrow v_{p h} / c_{h}$, where $v_{A p}$ and $c_{p, h}$ are the Alfvén speed based on the protons (which must be modified if we take into account a finite electron pressure) and ion sound speeds, respectively. It is useful to renormalize the phase speed to the Alfvén speed $v_{A p}$ and the $x$-coordinate to the proton inertial length $v_{A p} / \Omega_{p}$.

Figure 9 shows the variation of $\omega / k$ with $k$ for $c_{p}=0.3$, $c_{h}=0.1, \alpha=0.2, \mu=15$. The upper mode arises because of a bi-ion resonance. At short wavelengths this mode approaches the velocity which, in the case of a cold bi-ion plasma, is determined by the second condition in (42), i.e. 


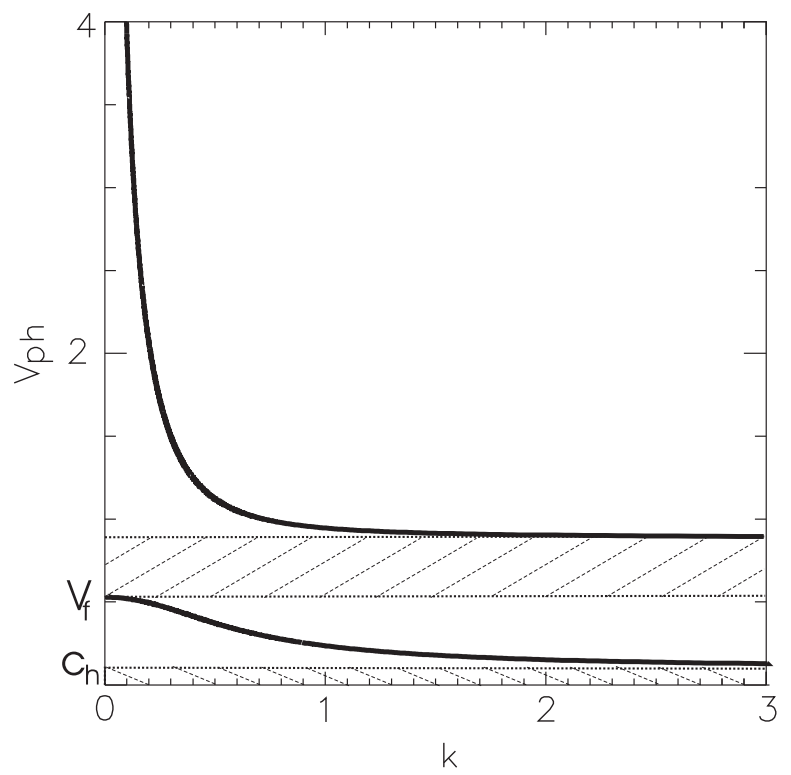

Fig. 9. Dispersion diagram of waves in bi-ion plasma with $c_{p}=$ $0.3, c_{h}=0.1, \alpha=0.2, \mu=15$ propagating transverse to the magnetic field. The hatched regions show where solitons may exist.

by the Alfvén speed modified by the density and charge ratios $\alpha q$. The hatched region below this velocity corresponds to where $k$ is imaginary and therefore indicates the region in which solitons may exist. The lower border of this region is the phase speed of another long wavelength mode $(k \rightarrow 0)$ which is determined by the expression (44) ("fast" speed for bi-ion plasma). The "fast" speed decreases with increasing $k$, approaching the heavy ion sound speed $c_{h}$ giving rise to a characteristic dispersion length scale which is a complicated function of the proton and heavy ion inertial lengths weighted by their characteristic speeds (and therefore may be called the compound inertial length). There is also another region, below $c_{h}$, in which $k$ is imaginary. However, in this "subsonic" regime, solitons cannot be constructed because critical points always intervene.

The existence condition which follows from the linear analysis is only a necessary one. Away from the neighbourhood of the initial point, the dynamics of the system becomes more complicated and cannot be described by the linearized Eq. (45). The behaviour of other terms in the RHS of Eq. (30) become important and the effects of critical Mach numbers appear. Although this is a complicated multi-parameter problem we have shown how it can be investigated for any given set of initial parameters. The results demonstrate the complex dependence between the soliton properties and the characteristic Mach numbers.

The case where the abundance of heavy ions is rather high ( $\alpha=n_{h o} / n_{p o}=0.2$, where $\alpha$ is the density ratio between the protons and the heavy ions), is typical for cometary environments (Goldstein et al., 1992) and in the inner region of the Martian magnetosheath (Dubinin et al., 1996a, b). It is worth noting that the main features of stationary solutions re- main essentially the same even if the heavy ions are a minor component, but the amplitudes of the stationary structures are correspondingly weaker.

Many important features of stationary flows can be elucidated by making use only of the conservation of total momentum, quasi-charge neutrality and zero electric current in the direction of wave propagation. This useful diagnostic of soliton structure has been successfully applied by McKenzie et al. (2001a) for the case of a cold bi-ion plasma, which revealed the qualitative structure of the flow and the magnetic field in a solitary-type pulse. In compressive solutions, the protons and the ions are decelerated along with the electrons. Due to differential streaming, a strong coupling between ion species arises and controls their relative motion. An interesting feature of the momentum hodograph is the existence of a second point (besides the initial point) at which all fluids have the same speed. The location of this point with respect to the initial point determines whether or not a soliton solution can be constructed. For example, the momentum hodograph tells us that a rarefaction soliton cannot be constructed (in supersonic flows) due to the absence (in the rarefaction branch) of the point where all species again reach the same speed.

However, the momentum hodograph alone does not determine the "equilibrium point" from which the system evolves back to the initial point. The problem of critical Mach numbers can also only be solved by analyzing the differential equation, which is highly nonlinear and a second order, but can be reduced to a first order differential equation by using the energy flux conservation. A detailed analysis of phase trajectories in the phase space $d u_{i} / d x, u_{i}$ is then described. Soliton solutions are possible in some specific regions of the three-dimensional phase space $M_{f}-M_{p}-M_{h}$, determined by the characteristic Mach numbers. In the limit of cold ions and electrons, solitons can be constructed if the flow speed exceeds the Alfvén speed based on the total ion mass and is less than the Alfvén speed based on the protons and reduced by a factor $1+\alpha q$, where $\alpha$ is the density ratio and $q$ is the charge of secondary ions. The solitons may have very different properties. There is an interesting class of solitons that contain an embedded rarefaction proton substructure. Within this rarefaction core, the protons may be reaccelerated almost up to the initial speed. A class of stationary solutions representing periodical nonlinear waves can also be constructed. However, the transition to the state with periodical waves can only be reached through a discontinuity.

The important feature of a bi-ion plasma with finite ion pressures is that in a broad range of ion-sonic Mach numbers $M_{p}, M_{h}$, critical points, where $d u_{p x} / d x \rightarrow \infty$, intervene before equilibrium points are reached. These critical Mach numbers significantly limit the range of flow speeds where smooth soliton or periodical solutions can be constructed. The relationship between the critical speeds of a bi-ion flow and the characteristic phase speeds of the system can be illuminated with the simpler case of ion-acoustic solitons in a proton-electron plasma using a gas-dynamical viewpoint (see McKenzie, 2001). In this case a necessary condition for 
the existence of a soliton is that the incoming proton speed exceeds the ion-acoustic speed of long wavelength waves $\left(\sqrt{k\left(T_{e}+T_{p}\right) / m_{p}}\right)$. However, the maximum strength is limited by the short wavelength speed $\left(\sqrt{k T_{p} / m_{p}}\right)$, because the flow is compressive and is driven towards the sonic point of the system where it is choked. In a bi-ion flow, such as the solar wind, in which the protons and alphas stream differentially, the concept of a short wavelength sonic point must be generalized (see McKenzie et al., 1993). In this case it follows that the "sonic point" in fact corresponds to locii in $\left(u_{p}, u_{\alpha}\right)$ space which is determined by the sound speeds of the heavy ions and protons, as well as their mass and density admixtures (composition). Similarly, in the problem here, a necessary condition for soliton existence is the requirement of "super-fast" flow at $k \rightarrow 0$ (see Fig. 9). Within the transition, the heavy ions and protons stream differentially and the "critical points" occur on "sonic locii" which correspond to the short wavelength dispersion equation for the system, including differential streaming.

Acknowledgements. E. Dubinin thanks the Deutsche Forschungsgemeinschaft and the Max-Planck-Gesellschaft for supporting this work by grants. The work was also supported by INTAS-ESA-9900066 grant.

\section{References}

Adlam, J. and J. Allen: The structure of strong collision-free hydromagnetic waves, Philos. Mag., 3, 448, 1958.

Dubinin E., Sauer, K., and Lundin, R., et al.: Plasma characteristics of the boundary layer in the martian magnetosphere, J. Geophys. Res., 101, 27 061, 1996a.

Dubinin E., Sauer, K., Lundin, R., Baumgärtel, K., and Bogdanov, A.: Structuring of the transition region (plasma mantle) of the martian magnetosphere, Geophys. Res. Lett., 23, 785, 1996 b.

Goldstein, B. E., Goldstein, R., and Neugebauer, M., et al.: Observations of plasma dynamics in the coma of P/Halley by the Giotto ion mass spectrometer, J. Geophys. Res., 97, 4121, 1992.

McKenzie, J. F.: The ion-acoustic soliton: A gas-dynamic viewpoint Physics of Plasma, submitted, 2001.

McKenzie, J. F., Marsch, E., Baumgärtel, K., and K. Sauer: Wave and stability properties of multi-ion plasmas with applications to winds and flows, Ann. Geophysicae, 11, 341, 1993.

McKenzie, J. F., Sauer, K., and Dubinin, E.: Stationary waves in a bi-ion plasma transverse to the magnetic field, J. Plasma Physics, 65, 197, 2001a.

McKenzie, J. F., Dubinin, E., and Sauer, K.: Nonlinear waves propagating transverse to the magnetic field, J. Plasma Physics, 65, 213, $2001 b$.

Motschmann U., Sauer, K., Roatsch, T., and McKenzie, J. F.: Subcritical milti-ion shocks, J. Geophys. Res., 96, 13 841, 1991.

Sagdeev, R. Z.: Cooperative phenomena and shock waves in collisionless plasma, Rev. Plasma Phys., English Transl. 4, 23, 1966.

Sauer, K., Bogdanov, A., and Baumgärtel, K.: Evidence of an ion composition boundary (protonopause) in bi-ion fluid simulations of solar wind mass loading, Geophys. Res. Lett., 21, 2255, 1994.

Sauer, K., McKenzie, J. F., and Dubinin, E.: Waves and nonlinear structures in bi-ion plasmas, in: Waves in Dusty, Solar and Space Plasmas, (Eds) Verheest, F., Goossens, M., Hellberg, M. A., and Bharuthram, R., 327, American Institute of Physics Conference Procedings Series, Woodhead, NY, USA, 2000.

Tidman, D. A. and Krall, N. A.: in Shock Waves in Collisionless Plasmas, Wiley-Interscince, New York, 1971. 